\title{
COVID-19 alters thinking and management in metabolic diseases
}

\section{Carel W. le Roux (1)}

Metabolic diseases emerged as important risk factors for severe COVID-19, but the mechanisms responsible remained unclear for some time. The severity of metabolic diseases was also associated with worse outcomes in patients with COVID-19, forcing clinicians to adjust their thinking on which patients with metabolic disease, but without COVID-19, to prioritize for treatment during and immediately after the pandemic.

When the first reports of a new emerging respiratory virus (SARS-CoV-2) reached us, we each generated an image of the patient groups who might be at risk. Very few of us could imagine that our hospitals' intensive care units (ICUs) would quickly fill with patients with COVID-19 who had underlying metabolic diseases such as type 1 and type 2 diabetes mellitus (referred to jointly as diabetes mellitus in this article) and obesity. Simple clinical observation of patients in ICUs who required mechanical ventilation revealed them to be younger than expected and often with obesity. The first published reports ${ }^{1}$ suggested a 'doseresponse curve' with those who had higher body weights being at most risk. This finding was quickly substantiated ${ }^{2}$ and triggered a new set of questions as ICU clinicians became accustomed to the challenges of mechanically ventilating patients with obesity. The prospect of having to use higher pressures owing to decreased chest wall compliance and greater transpulmonary pressures to facilitate sufficient oxygenation in those with acute lung injury (including the typical acute respiratory distress syndrome (ARDS)) and obesity was a considerable cause of concern. Paradoxically, these patients did not demonstrate the expected 'stiff lungs' or decreased lung compliance typical in patients with ARDS from other aetiologies ${ }^{3}$. It increasingly became apparent that early use of positive pressure ventilation, especially if not correctly applied, only increased the morbidity and mortality owing to a compounding ventilator-induced lung injury ${ }^{4}$. This finding led to a more conservative approach of permissive hypoxaemia and earlier non-invasive techniques to increase oxygenation by focusing on lung perfusion instead of ventilation as the primary objective ${ }^{4}$.

The game-changing explanation for why mechanical ventilation required reduced pressures came after detailed post-mortems were performed on seven patients with a BMI of $30 \pm 2.7 \mathrm{~kg} / \mathrm{m}^{2}$ (mean $\pm \mathrm{SEM}$ ) and established metabolic disease ${ }^{5}$. Instead of this breakthrough coming through sophisticated molecular techniques or after large scale randomized controlled trials, it was in fact the microscope that revealed pulmonary vascular endothelialitis, thrombosis and angiogenesis in patients who died from COVID-19. SARS-CoV-2 appeared to be amplifying existing damage in the microvascular bed due to the complications associated with diabetes mellitus and obesity. This knowledge improved treatments as we moved away from early mechanical ventilation and focused therapies around reducing inflammation and coagulability ${ }^{6}$.

A repetitive theme of the key advances in 2020 is old school approaches used by good physicians making solid clinical observations. Many of the advances did not need a scatter gun approach of multiple investigations followed by data mining, in the hope that new patterns would emerge. Again, bedside observations indicated new presentations of diabetes mellitus with diabetic ketoacidosis and hyperosmolarity, which required exceptionally high doses of insulin to control blood levels of glucose, a picture that did not quite fit with our current understanding of diabetes mellitus ${ }^{7}$. The emergence of this new form of diabetes mellitus suggested a bidirectional relationship between COVID-19 and metabolic diseases. Not only did metabolic disease potentiate the severity of COVID-19, but infection with the virus also revealed potential pre-existing metabolic frailty ${ }^{7}$. The main entry receptor for SARS-CoV-2 is proposed to be angiotensin-converting enzyme 2 (ACE2). These receptors are expressed in key metabolic organs and tissues, including pancreatic $\beta$-cells, adipose tissue, the small intestine and the kidneys ${ }^{7}$. The CoviDiab Project established a global registry of patients with diabetes mellitus related to COVID-19 to understand the extent and phenotype of new-onset diabetes mellitus defined by hyperglycaemia, confirmed COVID-19 and a negative history of diabetes mellitus. This registry provided another example of clinicians and scientists pulling together internationally during the COVID-19 pandemic to further knowledge that could result in better care for our patients.

It is well established that the worse the metabolic control the higher the risks of microvascular and macrovascular diseases in

\section{Key advances}

- Metabolic diseases emerged early in the pandemic as important risk factors for mechanical ventilation and mortality in patients with COVID-19 (REF. ${ }^{1}$ ).

- The mechanisms underlying increased mortality involve the microvascular bed, which helped our understanding of why patients with metabolic diseases were at increased risk 5 .

- A new form of diabetes mellitus was described, which suggests a bidirectional relationship between COVID-19 and metabolic diseases?

- Increased severity of metabolic diseases was implicated in worse outcomes from COVID-19 (REF. ${ }^{8}$ ).

- Clinicians had to adjust their thinking on which patients to prioritize for treatment during, but also immediately after, the COVID-19 pandemic ${ }^{10}$. 
patients with diabetes mellitus. We should not have been surprised that this observation was also true for the complications of COVID-19 in patients with diabetes mellitus ${ }^{8}$. The lack of this knowledge in the beginning of the pandemic caused confusion. Many clinicians reduced doses of medications or stopped certain drugs all together in patients with diabetes mellitus, as they were concerned that the medications themselves could enhance the virus' ability to cause harm ${ }^{9}$. However, the pendulums were constantly swinging and as evidence emerged that obesity was an independent risk factor for severe COVID-19 ${ }^{1}$, the focus was shifting towards type 2 diabetes mellitus and COVID-19. Type 1 diabetes mellitus was then brought into sharp focus by a series of papers showing it increases risk of death threefold ${ }^{8}$. Other diseases, such as chronic kidney disease with all its metabolic sequelae, were also identified as independent risk factors for severe COVID-19, again emphasizing the importance of a double hit (that is, adding COVID-19 to a system already affected by metabolic disease) on the microvascular bed of the lung, kidney and other organs ${ }^{5,6}$.

While we were learning about COVID-19, our usual hospital services were grinding to a halt. Patients already at high risk of complications due to diabetes mellitus, obesity or other metabolic diseases were deprioritized owing

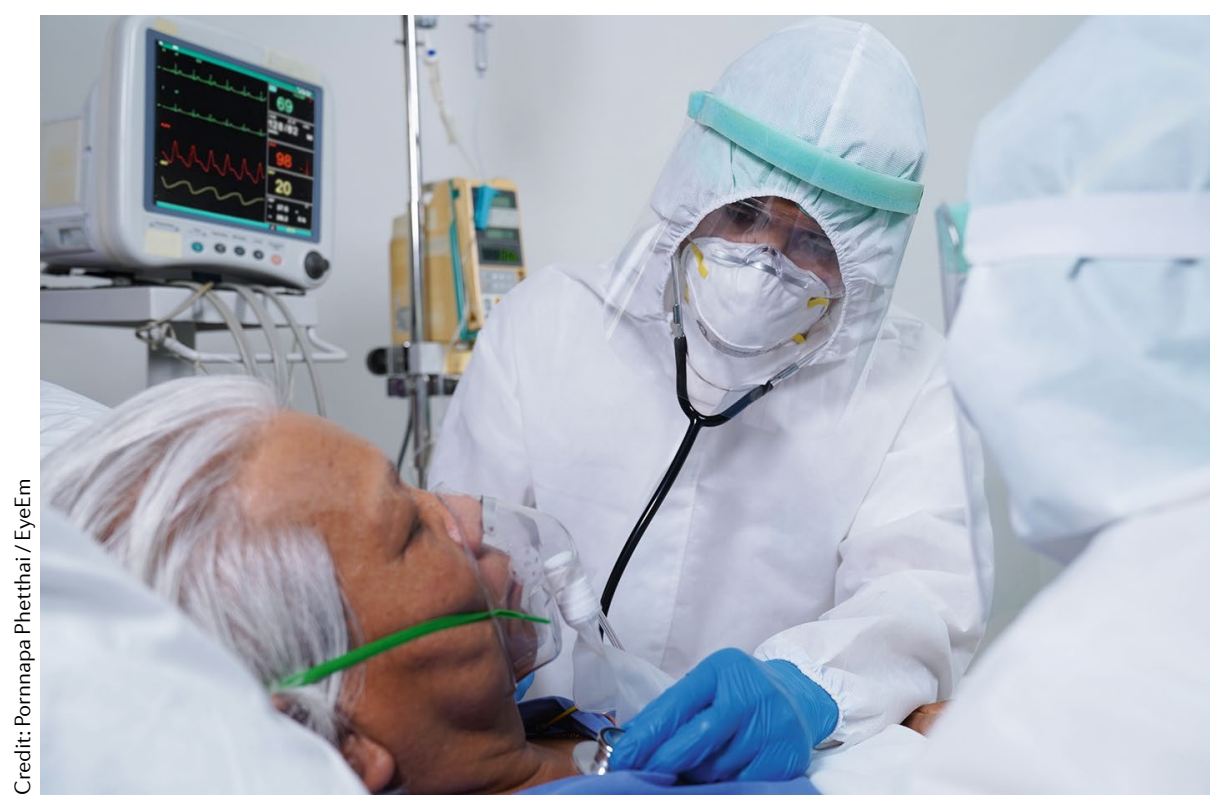

to a lack of understanding of what we were really dealing with. We had grown so accustomed to the large number of people with diabetes mellitus who died every year before the pandemic that we lost sight of the potential harm we might cause by not continuing their care. Obesity also came to prominence during the pandemic as an independent risk factor for severe complications of COVID-19, resulting in many viewing obesity as a disease and not the result of lifestyle choices for the first time ${ }^{10}$. With a lack of high-quality evidence, we reverted to gathering consensus among the 'great and the good'. Novel suggestions emerged regarding how patients with diabetes mellitus and obesity might be prioritized for treatment when resources are scarce during and after the COVID-19 pandemic ${ }^{10}$. Albeit that this method was the only way to guide practice, it might also represent a slippery slope. COVID-19 forced us to revert to clinical decision-making processes that were prominent in the mid-twentieth century: we used good clinical observation and older techniques of investigation to form opinions, but we then had to resort to clinical consensus without high-quality evidence to create guidelines. We should acknowledge that we have done the best we can, but we should now push forward to use the breakthroughs of 2020 and start re-applying higher standards for decision-making.
In conclusion, our thinking about SARS$\mathrm{CoV}-2$ has changed from it causing a respiratory disease towards now considering it a facilitator of complications of metabolic diseases. The COVID-19 pandemic alerted us again to the vulnerability of our patients with diabetes mellitus and obesity, by changing the image burnt into our memories of those who perished in our hospitals despite our best efforts. We should now redouble our efforts to improve our understanding of these metabolic diseases, not only to help patients through the COVID-19 pandemic, but also to reduce the morbidity and mortality of these diseases once COVID-19 has passed.

Carel W. le Roux (iD

Diabetes Complications Research Centre, Conway Institute, University College Dublin

Dublin, Ireland.

e-mail:carel.leroux@ucd.ie

https://doi.org/10.1038/s41574-020-00449-y

1. Simonnet, A. et al. high prevalence of obesity in severe acute respiratory syndrome coronavirus- 2 (SARS-CoV-2) requiring invasive mechanical ventilation Obesity 28, 1195-1199 (2020).

2. Caussy, C. et al. Prevalence of obesity among adult inpatients with COVID-19 in France. Lancet Diabetes Endocrinol. 8, 562-564 (2020).

3. Gattinoni, L., Coppola, S., Cressoni, M., Busana, M. $\&$ Chiumello, D. Covid-19 does not lead to a "typical" acute respiratory distress syndrome. Am. J. Respir. Crit. Care Med. 201, 1299-300 (2020).

4. Santamarina, M. G. et al. COVID-19: a hypothesis regarding the ventilation-perfusion mismatch. Crit. Care 24, 395 (2020)

5. Ackermann, M. et al. Pulmonary vascular endothelialitis, thrombosis, and angiogenesis in Covid-19. N. Engl. J. Med. 383, 120-128 (2020).

6. Pons, S., Fodil, S., Azoulay, E. \& Zafrani, L. The vascular endothelium: the cornerstone of organ dysfunction in severe SARS-CoV-2 infection. Crit. Care 24, 353 (2020).

7. Rubino, F. et al. New-onset diabetes in Covid-19. N. Engl. J. Med. 383, 789-790 (2020).

8. Barron, E. et al. Associations of type 1 and type 2 diabetes with COVID-19-related mortality in England: a whole-population study. Lancet Diabetes Endocrinol. 8 813-822 (2020).

9. Jarcho, J. A., Ingelfinger, J. R., Hamel, M. B., D'Agostino, R. B. Sr \& Harrington, D. P. Inhibitors of the renin-angiotensin-aldosterone system and Covid-19. N. Engl. J. Med. 382, 2462-2464 (2020).

10. Rubino, F. et al. Bariatric and metabolic surgery during and after the COVID-19 pandemic: DSS recommendations for management of surgical candidates and postoperative patients and prioritisation of access to surgery. Lancet Diabetes Endocrinol. 8, 640-648 (2020).

\section{Competing interests}

C.W.I.R. serves on advisory boards for Boehringer Ingelheim, GI Dynamics, Herbalife, Johnson\&Johnson, Keyron, Novo Nordisk and Sanofi. 